\title{
Molecular Docking and 3D-QSAR Studies on Substituted Imidazoles as Inhibitors of P38a MAP Kinase
}

\author{
MOHAN BABU JATAVATH, SABIHA FATIMA, SREE KANTH SIVAN and \\ VIJJULATHA MANGA*
}
Molecular Modeling and Medicinal Chemistry Group, Department of Chemistry, Nizam College, Osmania University, Basheerbagh, Hyderabad-500 001, India vijjulathamanga@gmail.com

Received 14 March 2013 / Accepted 30 April 2013

\begin{abstract}
Map kinases control many cellular events from complex programs, such as embryogenesis, cell differentiation, cell proliferation and cell death to short-term changes required for homeostasis and acute hormonal responses. Molecular docking and 3D-QSAR studies were performed on human P38 $\alpha$ MAP kinase inhibitors. Docked conformation obtained for each molecule was used as such for 3DQSAR analysis. Molecules were divided into training and test set randomly. PLS analysis was performed to obtain QSAR models using CoMFA and CoMSIA studies. Derived models showed good statistical reliability that is evident from their $\mathrm{q}^{2}{ }_{l o o}, \mathrm{r}^{2}$ and $\mathrm{r}_{\text {pred }}^{2}$ values. Information obtained from the 3D-QSAR helped us in optimization of lead molecule and design of novel potential inhibitors.
\end{abstract}

Keywords: P38 $\alpha$ Mitogen Activated Protein Kinase, Imidazoles, Comparative molecular field analysis, Comparative molecular similarity indices analysis, Docking

\section{Introduction}

Cellular responses to external stimuli involve the activation of several types of MitogenActivated Protein Kinase (MAPK) signalling pathways. MAPKs are a family of Serine/ threonine kinases that comprise three major sub groups, namely, ERK (Extracellular signalRegulated Kinase). p38 MAPK and JNK(c-Jun N-terminal Kinases) ${ }^{1}$. MAP Kinases are activated by protein kinase like tyrosine and threonine phosphorylation ${ }^{2}$. The phosphorylation motif Thr-Xaa-Tyr is located in the so called activation loop or T loop whose amino acid sequence varies among different MAPK subfamilies. Accordingly, there are different activating MAP Kinases that in most cases are specific for each subgroup of MAPKs. p38 MAPKs belong to mammalian Stress-activated MAPK family. The p38 MAPK subfamily plays important roles in cytokine production and in Stress response ${ }^{3}$. Recent reports have also demonstrated additional functions for p38 MAPKs, for example, in the inhibition of cell cycle progression, in developmental processes such as egg polarity and wing morphogenesis in Drosophila and in the differentiation of several vertebrate cell types including Neurons, Adipocytes and Myoblasts. The mammalian p38 MAPK families are activated by cellular stress including UV irradiation, heat shock, high Osmotic stress, Lipopolysaccharide, Protein 
synthesis inhibitors, Proinflammatory cytokines ${ }^{4,5}$ (such as IL-1 (Interleukin-1) and TNF-Alpha (Tumor Necrosis Factor-Alpha)) and certain Mitogens. Proinflammatory Cytokines such as IL (Interleukin) and TNF (Tumor Necrosis Factor) also stimulate p38 signalling. IL-1 (Interleukin-1) is a central regulator of the immune and inflammatory responses by which various inflammatory genes are induced. IL-1 signalling is known to involve PI3K, p38 MAPK and ERK. After IL-1 is bound to its receptor IL-1R (IL-1 Receptor), a complex is formed between the Type-1 Receptor and the Receptor Accessory Protein. The cytosolic proteins MyD88 (Myeloid Differentiation primary response gene-88) and TollIP (Toll-Interacting Protein) are recruited to this complex, where they function as Adaptors, recruiting IRAK1 (IL-1 Receptor-Associated Kinase-1) in turn. IRAK1, a Serine-threonine Kinase, activates and recruits TRAF6 (TNF Receptor-Associated Factors-6) to the IL-1 Receptor complex. Eventually, phosphorylated IRAK is ubiquitinated and degraded. TRAF6 signals through the TAB1 (TAK1 Binding Protein-1)/TAK1 (TGF-Beta-Activating Kinase-1) Kinases to activate MKKs, that further activates p38 MAPK. p38 is also activated by another Cytokine, TNF. Binding of the Receptor TNFR $1^{6}$ to TNF-Alpha results in conformational changes in the receptor's intracellular domain, resulting in rapid recruitment of several cytoplasmic death domain - containing adaptor proteins via homophilic interaction with the death domain of the receptor. In general, p38 appears to play a major role in Apoptosis, Cytokine production, Transcriptional regulation ${ }^{7}$, Cytoskeletal reorganization has been causally implicated in Sepsis, Ischemic Heart disease, Arthritis ${ }^{8}$, Human Immunodeficiency Virus infection and Alzheimer's disease ${ }^{9}$. A series of substituted imidazoles are identified for the regulation of p38 MAP Kinases overstimulation.

\section{Experimental}

A total of 40 molecules ${ }^{10}$ available with reported $\mathrm{IC}_{50}$ values for inhibition of P38 MAP Kinase, these values were converted to corresponding $\mathrm{pIC}_{50}$ values (Table 1). Data set was divided into training and test set randomly. All molecular modeling calculations were performed on a Linux operating system. Crystal structure of p38 $\alpha$ MAP Kinase bound with Monocyclic Pyrazolone inhibitor (pdb id: 1 YWR) ${ }^{10}$ was downloaded from the protein data bank. GLIDE $5.6^{11}$ was used for molecular docking. Protein was prepared using protein preparation module applying default parameters. A grid was generated around active site of p38 $\alpha$ MAP Kinase ${ }^{12-14}$ catalytic domain by selecting the co-crystallized ligand, with receptor van der Waals scaling for non polar atoms as $0.9,{ }^{15}$ which makes protein site "roomier" by moving back the surface of non polar regions of protein and ligand. This kind of adjustments emulate to some extent the effect of breathing motion to protein site, it is a kind of giving breathing to the receptor, this approach softens active site region of the receptor making it flexible ${ }^{16}$. Forty molecules were built using maestro build panel and prepared by LigPrep application. Structure of molecules, their $\mathrm{IC}_{50}$ and $\mathrm{pIC}_{50}$ are given in Table 1.

LigPrep produces low energy conformer of the ligand using the MMFF94s force field. These molecules were docked into the grid generated from p38 $\alpha$ MAP Kinase protein structures using standard precision docking mode. Co-crystallized ligand was also docked and its RMSD was calculated to validate the docking process (Figure 1(a)).

Dock pose of each ligand docked into the protein active site was analyzed for their hydrogen bond interactions with the receptor (Figure 1(b)). Analysis of dock poses of all molecules showed similar hydrogen bond interactions with Met 109 an active site residue. Overlay of the most promising poses (best GLIDE Score along with most physiologically similar positions) has been taken directly into $3 \mathrm{D} \mathrm{QSAR}^{17,18}$ analysis in such a way QSAR is taken a step beyond what is usually done in such analysis. Overlay of dock pose of each ligand is shown in Figure 2. 

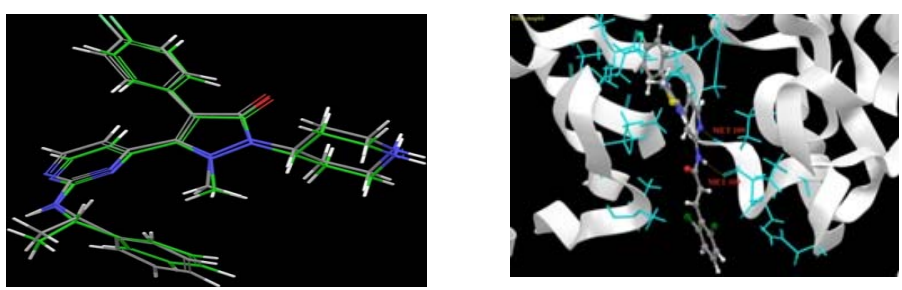

Figure 1(a). Superimposition of crystal structure pose (green) on docked pose (elemental) of co-crystallized ligand. RMS deviation is $0.3821 \AA$; (b) Dock pose of most active molecule 33 showing H-bond interactions with Meth109 of p38MAPK

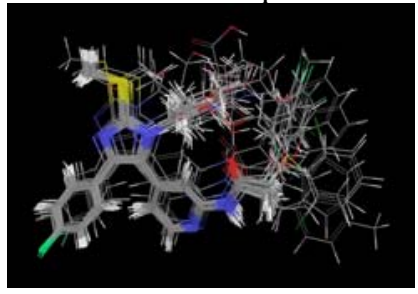

Figure 2. Docking based alignment of data set molecules

Table 1. Structure of Imidazole Molecules along with their $\mathrm{IC}_{50}, \mathrm{pIC}_{50}$, predicted $\mathrm{pIC}_{50}$ from CoMFA and CoMSIA

\begin{tabular}{llllllll} 
& \\
\hline
\end{tabular}




\begin{tabular}{|c|c|c|c|c|c|c|}
\hline 8 & & 0.44 & 9.3565 & 9.273 & 9.399 & -8.628 \\
\hline $9 *$ & & 0.33 & 9.4814 & 9.349 & 9.573 & -7.991 \\
\hline 10 & & 1.62 & 8.7904 & 8.838 & 8.788 & -8.409 \\
\hline $11 *$ & & 0.77 & 9.1135 & 9.210 & 8.988 & -9.281 \\
\hline 12 & & 1.14 & 8.9430 & 9.008 & 8.933 & -9.542 \\
\hline $13^{*}$ & & 1.57 & 8.8041 & 9.195 & 9.465 & -8.250 \\
\hline 14 & & 0.060 & 10.2218 & 10.176 & 10.229 & -8.499 \\
\hline $15^{*}$ & & 0.28 & 9.5528 & 10.059 & 9.928 & -8.687 \\
\hline 16 & & 0.78 & 9.1079 & 8.789 & 9.030 & -8.783 \\
\hline $17 *$ & & 0.79 & 9.1023 & 8.848 & 9.647 & -9.092 \\
\hline 18 & & 0.40 & 9.3979 & 9.393 & 9.533 & -8.589 \\
\hline $19^{*}$ & & 0.57 & 9.2441 & 9.099 & 10.074 & -8.330 \\
\hline 20 & $-\mathrm{CH}_{3}-\mathrm{CH}_{2}-\mathrm{CH}_{2}-\mathrm{Cl}$ & 0.017 & 10.7695 & 10.887 & 10.748 & -8.155 \\
\hline 21 & $\begin{array}{c}-\mathrm{CH}_{3}-\mathrm{CH}_{2}-\mathrm{CH}_{2-} \\
\mathrm{CH}_{2}-\mathrm{Cl}\end{array}$ & 0.18 & 9.7447 & 9.691 & 9.908 & -7.668 \\
\hline 22 & $-\mathrm{CH}_{3}-\mathrm{CH}_{2}-\mathrm{CH}_{2}-\mathrm{Br}$ & 0.027 & 10.5686 & 10.420 & 10.426 & -8.152 \\
\hline 23 & $-\mathrm{CH}_{3}-\mathrm{CH}_{2}-\mathrm{CH}_{2}-\mathrm{I}$ & 0.019 & 10.7212 & 10.759 & 10.698 & -8.146 \\
\hline \multirow{2}{*}{ Compound } & \multirow{2}{*}{$\mathrm{R}^{1}$} & \multirow{2}{*}{$\mathrm{R}^{2}$} & \multirow{2}{*}{$\begin{array}{l}\mathrm{IC}_{50} \\
\mu \mathrm{M}\end{array}$} & \multicolumn{2}{|c|}{ Pred. pIC ${ }_{50}$} & Dock \\
\hline & & & & CoMFA & CoMSIA & scores \\
\hline 24 & & & 0.17 & 9.7695 & 9.753 & 9.907 \\
\hline 25 & & & 0.49 & 9.3098 & 9.330 & 9.129 \\
\hline
\end{tabular}




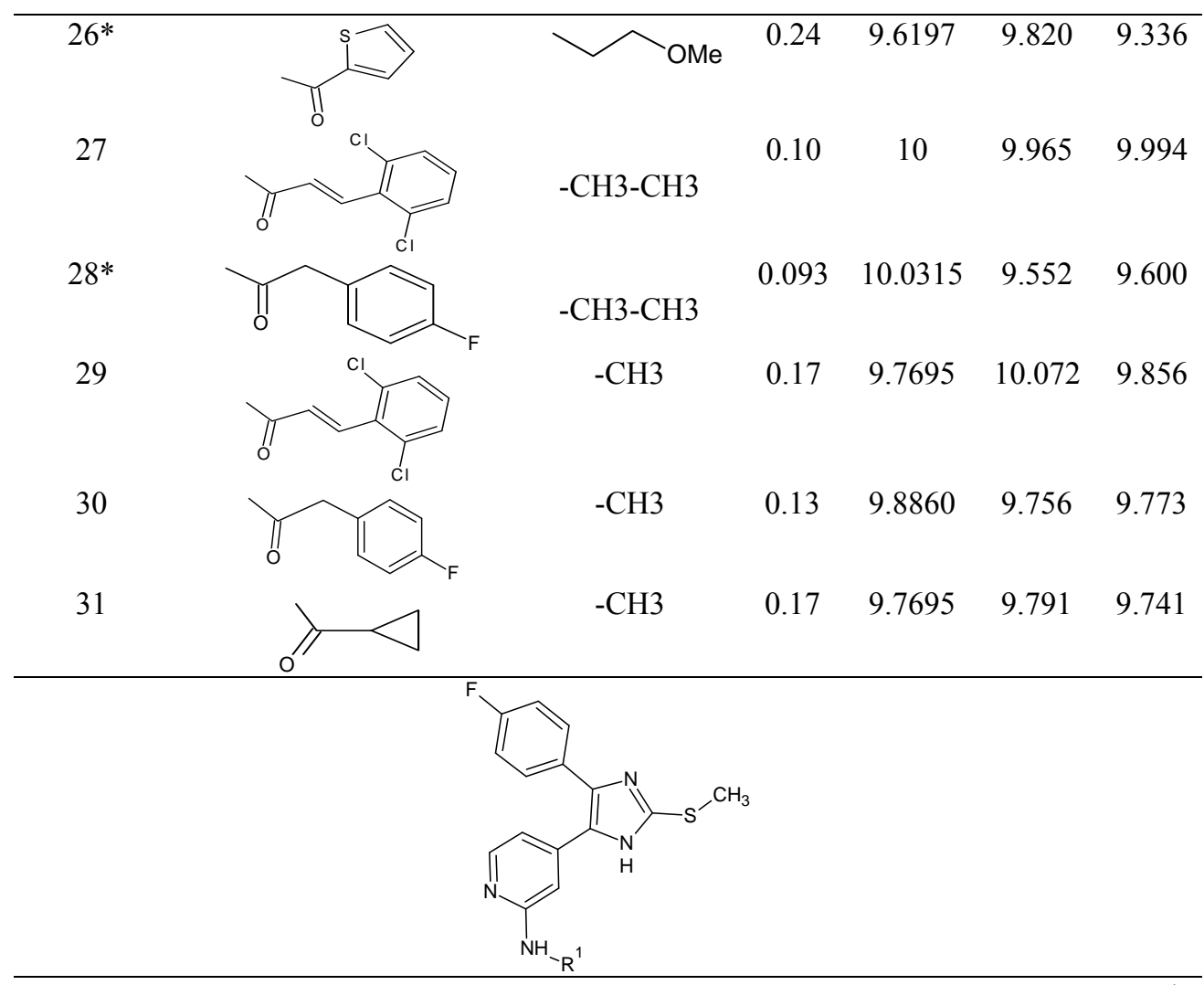

\begin{tabular}{|c|c|c|c|c|c|c|}
\hline Compound & $\mathrm{R}^{1}$ & $\mathrm{IC}_{50} \mu \mathrm{M}$ & $\mathrm{pIC}_{50}$ & \multicolumn{2}{|c|}{ Pred. pIC 50} & $\begin{array}{c}\text { Dock } \\
\text { scores }\end{array}$ \\
\hline $32 *$ & & 0.032 & 10.4948 & 10.997 & 10.942 & -6.917 \\
\hline 33 & & 0.003 & 11.5228 & 11.227 & 11.403 & -6.990 \\
\hline 34 & & 0.009 & 11.0457 & 10.967 & 10.974 & -8.899 \\
\hline 35 & & 0.014 & 10.8538 & 10.258 & 10.073 & -8.770 \\
\hline $36^{*}$ & & 0.10 & 10 & 9.690 & 9.780 & -8.377 \\
\hline 37 & & 0.16 & 9.7958 & 10.997 & 10.942 & -8.736 \\
\hline
\end{tabular}




\begin{tabular}{llllllll}
\hline $38 *$ & 0.053 & 10.2757 & 10.352 & 10.286 & -8.516 \\
40 & 0.027 & 10.5686 & 10.870 & 10.802 & -8.008 \\
\hline & 0.013 & 10.8860 & 10.867 & 10.843 & -8.226 \\
\hline
\end{tabular}

*test set molecules

Molecules were imported into SYBYLX-1.2 molecular modeling program package ${ }^{19}$ Gasteiger-Huckel $^{20}$ charges were assigned. Standard Tripos force fields were employed for CoMFA $^{21,22}$ and CoMSIA ${ }^{23}$ analysis. A 3 D cubic lattice of dimension $4 \AA$ in each direction with each lattice intersection of regularly spaced grid of $2.0 \AA$ was created. Steric and electrostatic parameters were calculated in CoMFA fields while hydrophobic, acceptor and donor parameters in addition to steric and electrostatic were calculated in CoMSIA fields at each lattice. $\mathrm{A} \mathrm{sp}^{3}$ carbon atom was used as a probe atom to generate steric (Lennard-Jones potential) field energies and a charge of +1 to generate electrostatic (Coulombic potential) field energies. A distance dependent dielectric constant of 1.00 was used. Steric and electrostatic fields were truncated at $+30.00 \mathrm{kcal} / \mathrm{mol}$.

A partial least squares regression was used to generate a linear relationship that correlates changes in the computed fields, with changes in corresponding experimental values of biological activity $\left(\mathrm{pIC}_{50}\right)$ for the data set of ligands. Forty molecules were divided into training and test set. Biological activity values of ligands were used as dependent variables in PLS statistical analysis. Column filtering value(s) was set to $2.0 \mathrm{kcal} / \mathrm{mol}$ to improve the signal-to-noise ratio by omitting those lattice points whose energy variations were below the threshold. Cross-validations were performed by leave-one-out (LOO) procedure to determine

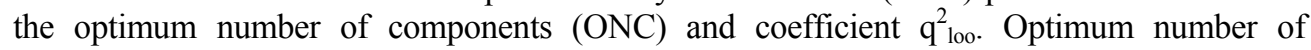
components obtained is then used to derive the final QSAR model using all the training set compounds with non-cross validation and to obtain conventional correlation coefficient $\left(\mathrm{r}^{2}\right)$. To validate CoMFA and CoMSIA derived models, predictive ability for the test set of compounds (expressed as $r_{\text {pred }}^{2}$ ) was determined by using the following equation

$$
r_{\text {pred }}^{2}=(\mathrm{SD}-\mathrm{PRESS}) / \mathrm{SD}
$$

$\mathrm{SD}$ is a sum of squared deviations between biological activities of test set molecules and mean activity of the training set. PRESS is sum of squared deviation between observed and predictive activities of the test set molecules.

\section{Results and Discussion}

P38 $\alpha$ MAP kinase inhibitors, shown in Table. 1 along with $\mathrm{IC}_{50}$ and $\mathrm{pIC}_{50}$ values, were docked into the active site; they showed two hydrogen bond interactions with the active site residues. Accuracy of docking protocol was evaluated by redocking the crystal structure ligand and its root mean square deviation from the experimental binding mode determined by $\mathrm{X}$-ray crystallography was calculated, and it gave a value of $0.731 \AA$. Molecules having specific hydrogen bond interactions with P38 $\alpha$ MAP kinase binding site of the catalytic domain in the enzyme, pyridine NH and NH of amide bond acts as a hydrogen bond donor to Met 109 were considered. 3D QSAR CoMFA and CoMSIA analysis were carried out on the reported inhibitors. Molecules having precise $\mathrm{IC}_{50}$ values were selected and those that did not show interactions with the protein active site (via docking) were removed from the data set. 
A set of 40 molecules were used for derivation of model, these were divided into training and test set of 28 and 12 respectively. CoMFA and CoMSIA statistical analysis are summarized in Table 2.

Table 2. PLS result summary

\begin{tabular}{ccc}
\hline \multicolumn{3}{c}{ Statistical parameters } \\
\hline CoMFA & CoMSIA \\
\hline Number of molecules in training set & 28 & 28 \\
Number of molecules in test set & 12 & 12 \\
ONC & 5 & 7 \\
SEE & 0.148 & 0.104 \\
$\mathrm{q}^{2}$ loo & 0.553 & 0.636 \\
$\mathrm{r}^{2}$ ncv & 0.972 & 0.987 \\
$\mathrm{r}^{2}$ pred & 0.544 & 0.632 \\
F value & 152.741 & 225.611 \\
Steric & & \\
Electrostatic & $61.6 \%$ & $16.0 \%$ \\
Hydrophobic & $38.4 \%$ & $16.7 \%$ \\
Donor & - & $27.5 \%$ \\
Acceptor & - & $20.5 \%$ \\
\hline
\end{tabular}

$\mathrm{q} 2_{1 \mathrm{oo}}=$ Cross-validated correlation coefficient by leave one out method, $\mathrm{r}_{\text {ncv }}^{2}=$ conventional correlation coefficient, $\mathrm{ONC}=$ optimum number of components, $\mathrm{SEE}=$ standard error of estimate, $\mathrm{F}=$ Fisher test value, $\mathrm{r}_{\text {pred }}^{2}=$ cross-validated correlation coefficient on test set.

Statistical data shows $\mathrm{q}_{\text {loo }}^{2}$ of 0.553 and 0.636 , non cross-validated correlation coefficients $\mathrm{r}_{\text {ncv }}^{2}$ of 0.972 and 0.987 respectively for CoMFA and CoMSIA. The $\mathrm{q}^{2}$ loo and $\mathrm{r}^{2}$ values indicate a good internal predictive ability of these models. To test predictive ability of the models, test set molecules excluded from the model generation were used. Predictive correlation coefficient $\mathrm{r}_{\text {pred }}^{2}$ of 0.544 for CoMFA and 0.632 for CoMSIA respectively indicates good external predictive ability of the models.

Scatter plot for actual and predicted $\mathrm{pIC}_{50}$ values for training and test set of CoMFA and CoMSIA studies are shown in Figure 3.

\section{Contour analysis}

To visualize information content of the derived 3D QSAR Models, CoMFA and CoMSIA contour maps were generated. Contour plots are the representation of lattice points and difference in the molecular field values, at lattice points, strongly connected with difference in receptor binding affinity. Molecular fields define favourable or unfavourable interaction energies of aligned molecules with a probe atom traversing across lattice plots suggest modification required to design new molecules. Contour maps of CoMFA denote region in the space where molecules would favourably or unfavourably interact with the receptor, while CoMSIA contour maps denote these areas within the specified region where presence of a group with a particular physicochemical activity binds to the receptor. CoMFA and CoMSIA results were graphically interpreted by field contribution maps using 'STDEV COEFF' field type. All contours represented default 80 and 20\% level contribution for favoured and disfavoured regions. Molecule 33, the most potent molecule among the series was displayed on the map in aid of visualization and molecule 5, least active are discussed using these contours. 

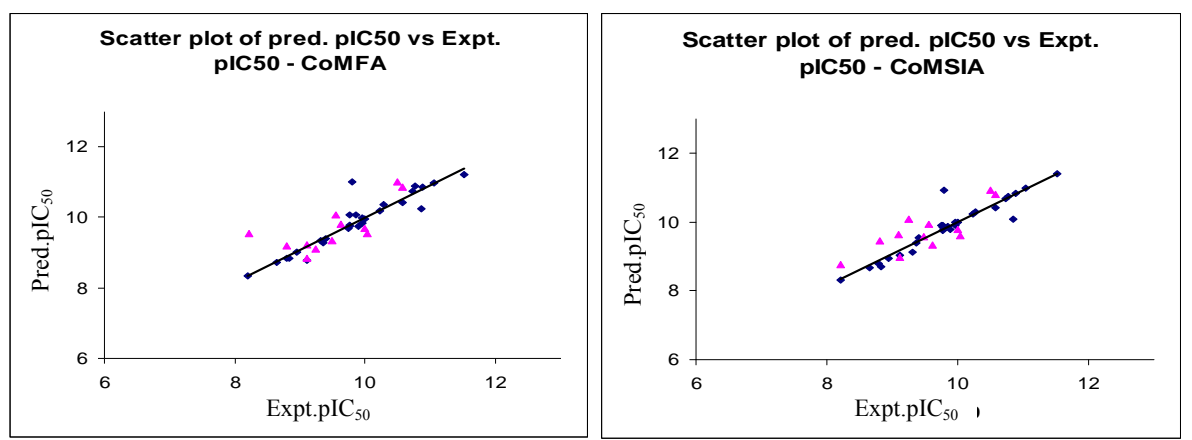

Figure 3. Scatter plot of predicted vs. experimental pIC50 values (test set is represented as triangles)

Figure 4 (a) and (b) shows contour maps for CoMFA steric fields of most active and least active molecules, green and yellow contours represent favoured and disfavoured regions respectively. In most active molecule 33, large green contour is seen above one of the chloro group in dichloro benzene ring suggesting substitution with bulky group at this position will increase the activity. A yellow contour is seen near the imidazole ring suggesting bulky substituents in this area will significantly decrease the biological activity, least active molecule 5, having 2-propyl-1,3-diol is intruding into yellow contour indicating its decreased activity. CoMSIA steric contours are similar to CoMFA (shown in Figure 4 (c) and (d)) apart from a large green contour on the imidazole group suggesting increase in bulkiness on the substituents present will favour enhanced biological activity. In case of least active molecule the substituent on the imidazole ring is extending towards the yellow contour hence decrease in activity is observed in molecule 5 .

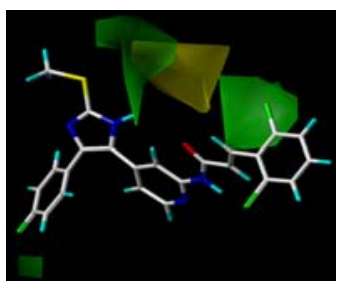

(a)

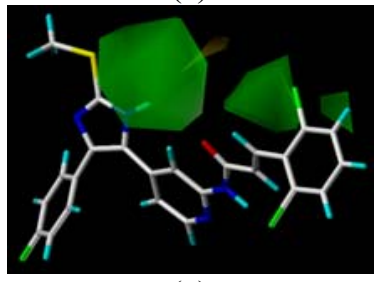

(c)

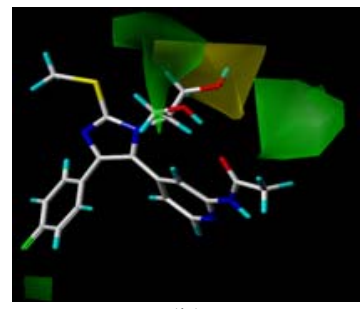

(b)

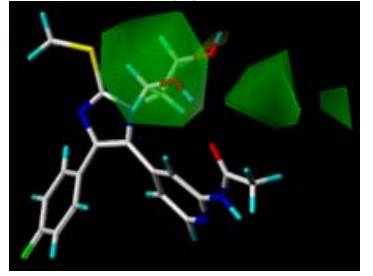

(d)

Figure 4. $(a, b)$ COMFA Steric fields contour map illustrating steric features in combination with (a)best active molecule 33 and (b) least active molecule 5. Green contours represent favourable bulky group substitution at that point while yellow regions are disfavourable for activity. (c, d) COMSIA Steric fields contour maps illustrating steric features in combination with (c) best active molecule 33 and (d) least active molecule 5. Green contours represent favourable bulky group substitution at that point while yellow regions are disfavourable for activity 
Figure 5 (a) and (b) depicts CoMFA electrostatic contour for most active and least active molecules. Red contour present at one of the imidazole ring nitrogen suggest that more electronegative atoms or groups at this position will significantly improve biological activity. For least active molecule red contours are away from the electronegative groups and one of the $\mathrm{OH}$ group of propane diol attached to the imidazole ring is encroaching the electropositive contour, self explaining its reduced activity.

Figure 5 (c) and (d) CoMSIA electrostatic contour for most and least active molecules. In most active molecule 33, NH of amide group is penetrating into a large red contour and more electronegative atom chlorine on benzene ring is very near to the red contour that are responsible for its higher activity. In least active molecule 5 the methyl group is penetrating into the red contour and $\mathrm{OH}$ group of propanediol attached to the imidazole ring is towards the electropositive contour that is indicative of the molecules reduced activity.

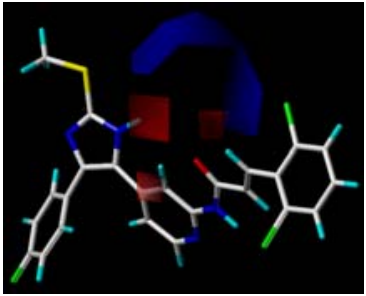

(a

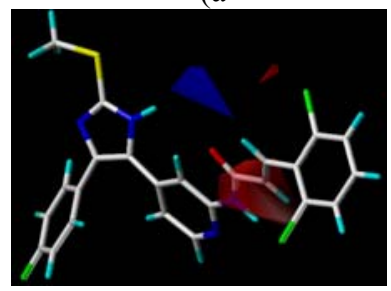

(c)

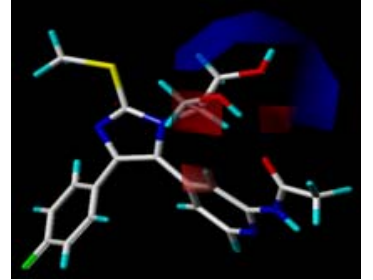

(b)

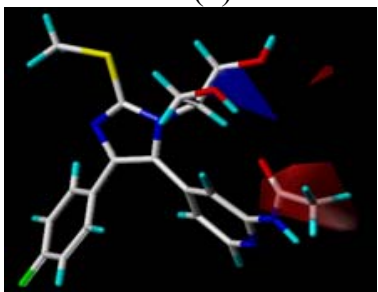

(d)

Figure 5. $(a, b)$ COMFA electrostatic fields contour maps illustrating electrostatic features in combination with (a) best active molecule 33 and (b) least active molecule 5 . Figure 5 (c, d) COMSIA electrostatic fields. (c) Contour maps illustrating electrostatic features in combination with best active molecule 33 and (d) Contour maps illustrating electrostatic features in combination with least active molecule 5. Red contours indicate negative charge favouring activity; where as blue contours indicate positive charge favouring activity

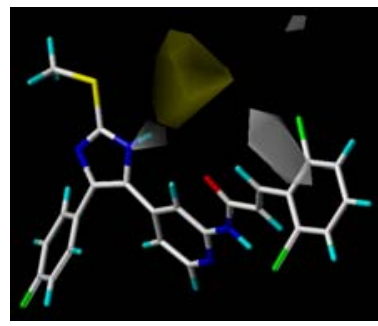

(a)

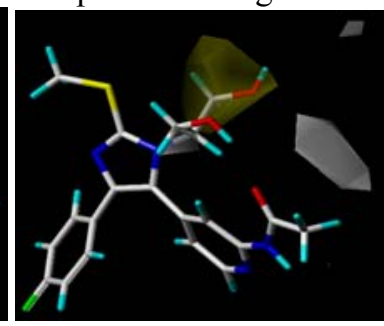

(b)

Figure 6. COMSIA hydrophobic fields.(a)contour maps illustrating hydrophobic features in combination with best active molecule 33 and (b) contour maps illustrating hydrophobic features in combination with least active molecule 5. Yellow contour represent hydrophobic favoured region, white indicates the hydrophilic favoured regions. 
Hydrophobic fields are represented in Figure 6, yellow and white contour highlighted areas, where hydrophobic and hydrophilic groups are preferred. A large white contour is seen in the most active molecule 33, near to the dichlorobenzene ring indicating that bulkier hydrophilic groups will increase the activity and a small white contour is there on imidazole nitrogen responsible for more activity of the molecule 33. In case of least active molecule the hydrophilic hydroxyl group is encroached the big yellow contour resulting in decreased activity.

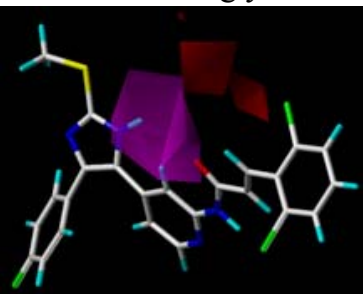

(a)

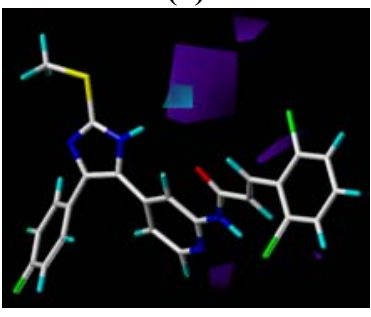

(c)

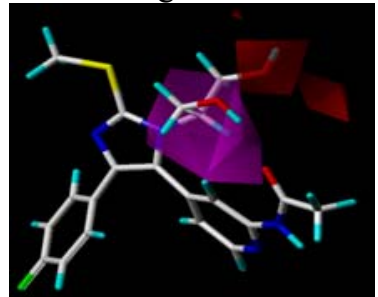

(b)

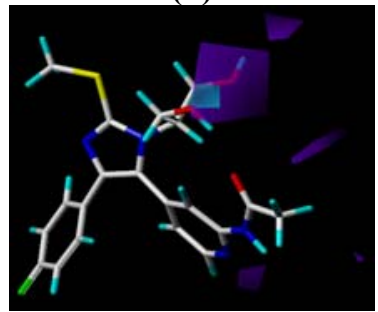

(d)

Figure 7. COMSIA hydrogen bond acceptor and donor fields (a) and (c) contour maps illustrating acceptor \& donor features in combination with best active molecule 33 (b) and (d) contour maps illustrating acceptor $\&$ donor features in combination with least active molecule 5

Figure 7 represents Hydrogen bond acceptor contours in magenta and red, while donor interactions are shown in cyan and purple for favoured and disfavoured respectively. For molecule 33 an acceptor favoured magenta contour is observed near $\mathrm{NH}$ of imidazole and near the carbonyl of peptide bond and a donor disfavoured purple contour is observed in the same region in figure 7c indicating the increase in activity. For the least active molecule 5 in figure $7 \mathrm{~d}, \mathrm{OH}$ a donor group on the side chain is towards the donor disfavoured (purple contour) region explain its decreased activity.

\section{Designed molecules}

Detailed contour analysis empowered us to identify structural requirements for observed inhibitory activity. New molecules were designed based on these structural requirements. Likewise, introduction of a hydrogen bond acceptor or hydrophilic group on benzene ring adjacent to the amide linkage increasing the affinity between the receptor and ligand, as shown in Figure 8.

New molecules designed were docked into the active site and they showed similar interactions with comparable dock score and predicted activity with respect to the most active molecule 33. Figure 8 shows dock pose of newly designed molecule N1, that shows three hydrogen bond interactions with the active site aminoacids Lys53, Met110 and Asp112, hence increasing the binding affinity. Predicted $\mathrm{pIC}_{50}$ values were calculated and found to be better, structures of newly designed molecules and their predicted $\mathrm{pIC}_{50}$ are given in Table 3. 


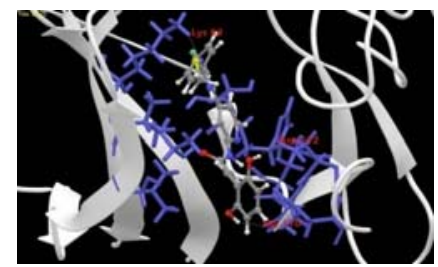

Figure 8. Dock pose of newly designed molecule N1 in the active site shows three hydrogen bond interactions with amino acid Lys53, Met110 and Asp112

Table 3. Structures of newly designed molecules with predicted $\mathrm{pIC}_{50}$ values

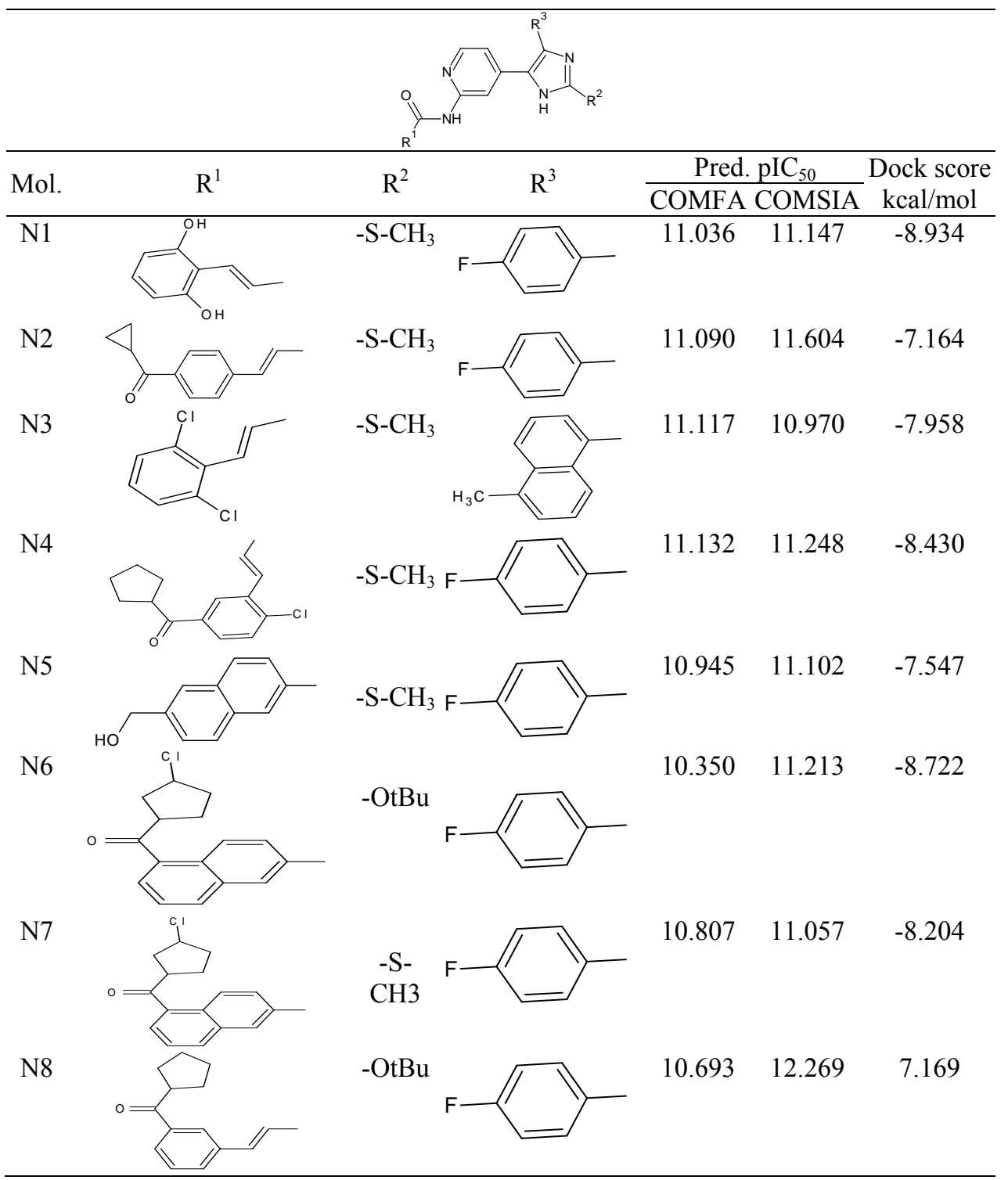




\section{Conclusion}

Molecular docking based 3D-QSAR studies are widely used tools for understanding binding modes of the molecule to the enzymatic receptors and rationalize the structural requirements for the inhibitory activity of the molecules. CoMFA and CoMSIA methodologies were used to build models for p38 $\alpha$ MAP kinase inhibitory activity of imidazole derivatives. The generated models have statistical reliability that is evident from high $\mathrm{r}^{2}$ and $\mathrm{q}^{2}$ values for all the models. Based on detailed contour map analysis, improvement in p38 $\alpha$ MAP kinase binding affinity has been achieved by substitutional modification at the benzene and pyridine rings. The designed molecules based on these parameters showed better predictive activity than reference molecules, this indicates QSAR models generated have good predictive ability to design potent inhibitors. These molecules can be synthesized to generate a greater number of molecules with required pharmacokinetics for further clinical studies.

\section{Acknowledgment}

We gratefully acknowledge support for this research from Department of Science and Technology, New Delhi, India, University Grants Commission, New Delhi, India and Department of chemistry, Nizam College, Hyderabad, India. We also acknowledge Schrödinger Inc. for GLIDE software, Tripos Inc. for SYBYLX-1.2.

\section{References}

1. Gary L J and Razvan L, Science, 2002, 298, 1911-1922.

2. Joël R, Shashi G, Jeffrey S R, Martin D, Jiahuai H and Richard J U and Roger J D, $J$ Bio Chem., 1995, 270(13), 7420-7426; DOI:10.1074/jbc.270.13.7420.

3. John C L and Peter R Y, J Leukocyte Biol., 1996, 59(2), 152-157.

4. Veronique B, Zheng-Gang L, Brydon B, Nobutaka S, Ying X and Michael Karin, Genes Dev., 1999, 13(10), 1297-1308.

5. Guan Z, Buckman S Y, Pentland A P, Templeton D J and Morrison A R, J Biol Chem.,1998, 273(21), 12901-12098; DOI:10.1074/jbc.273.21.12901.

6. Antony S, Soren B and Steven C L, Trend Immun.,.2006, 27(1), 40-48.

7. $\quad$ Tyler Z and Jiahuai H, Cell Res., 2005, 15(1), 11-18; DOI:10.1038/sj.cr.7290257.

8. Foster M L, Halley F and Souness J E, Drug News Perspect., 2000, 13(8), 488.

9. Kenneth H, Robert A F, Nai-Ying Z, Raha N, Kent A R, Xuan N, Quentin N P, Charles A S, James G and William R M, J Neurochem., 1999, 72(5), 2053-2058; DOI:10.1046/j.1471-4159.1999.0722053.x.

10. Stefan L, Dominik H, Thomas S, Claudia Bracht, Kathrin Ruff, Verena Schattel, Wolfgang Albrecht and Pierre Koch, Bioorg Med Chem Lett., 2010, 20(22), 66716675; DOI:10.1016/j.bmcl.2010.09.012.

11. Schrödinger, LLC, New York, Glide Version 5.6, 2010.

12. Hale K K, Trollinger D, Rihanek M and Manthey C L, J Immuno., 1999, 162(7), 4246-4252.

13. Allen M, Svensson L, Roach M, Hambor J, McNeish J and Gabel C A, J Exp Med., 2000, 191(5), 859-870; DOI:10.1084/jem.191.5.859.

14. Fearns C, Kline L, Gram H, Di Padova F, Zurini M, Han J and Ulevitch R J, J Leukocyte Biol., 2000, 67(5), 705-711.

15. Friesner R A, Banks J L, Murphy R B, Halgren T A, Klicic J J, Mainz D T, Repasky M P, Knoll E H, Shelley M, Perry J K, David Shaw E, Francis P and Shenkin P S, J Med Chem., 2004, 47(7), 1739-1749; Dol: 10.1021/jm0306430. 
16. Taverna D M and Goldstein R A, Proteins, 2002, 46(1), 105-109; DOI: $10.1002 /$ prot.10016.

17. Ajit P Z, Ashok L G, Devanand B S and Vithal M K, J Chem Inf Model., 2007, 47(2), 635-643; DOI:10.1021/ci6004367.

18. Vadlamudi S M and Kulkarni V M, Int Electron J Mol Des., 2004, 3(9), 586-609.

19. Sybyl-X1.2, Tripos Associates, St. Louis (MO), 2010; www.tripos.com/sybyl

20. Gasteiger J and Marsili M, Tetrahedron, 1980, 36(22), 3219-3228; DOI:10.1016/0040-4020(80)80168-2.

21. Cramer R D III, Patterson D E and Bunce J D, J Am Chem Soc., 1988, 110(18), 5959-5967; DOI:10.1021/ja00226a005.

22. Cramer R D III, Patterson D E, Ildiko E. Frank and Bunce J D, Quant Struct Act Relat., 1988, 7(1), 18-25; DOI:10.1002/qsar.19880070105.

23. Klebe G, Abraham U and Mietzner T, J Med Chem., 1994, 37(24), 4130-4146; DOI: $10.1021 / \mathrm{jm} 00050 \mathrm{a} 010$. 